\title{
Óleos essenciais utilizados no tratamento de neuralgias: Uma revisão sistemática
}

\author{
Essential oils used in the treatment of neuralgia: A systematic review \\ Aceites esenciales utilizados en el tratamiento de las neuralgias: Una revisión sistemática
}

Recebido: 03/04/2021 | Revisado: 11/04/2021 | Aceito: 14/04/2021 | Publicado: 27/04/2021

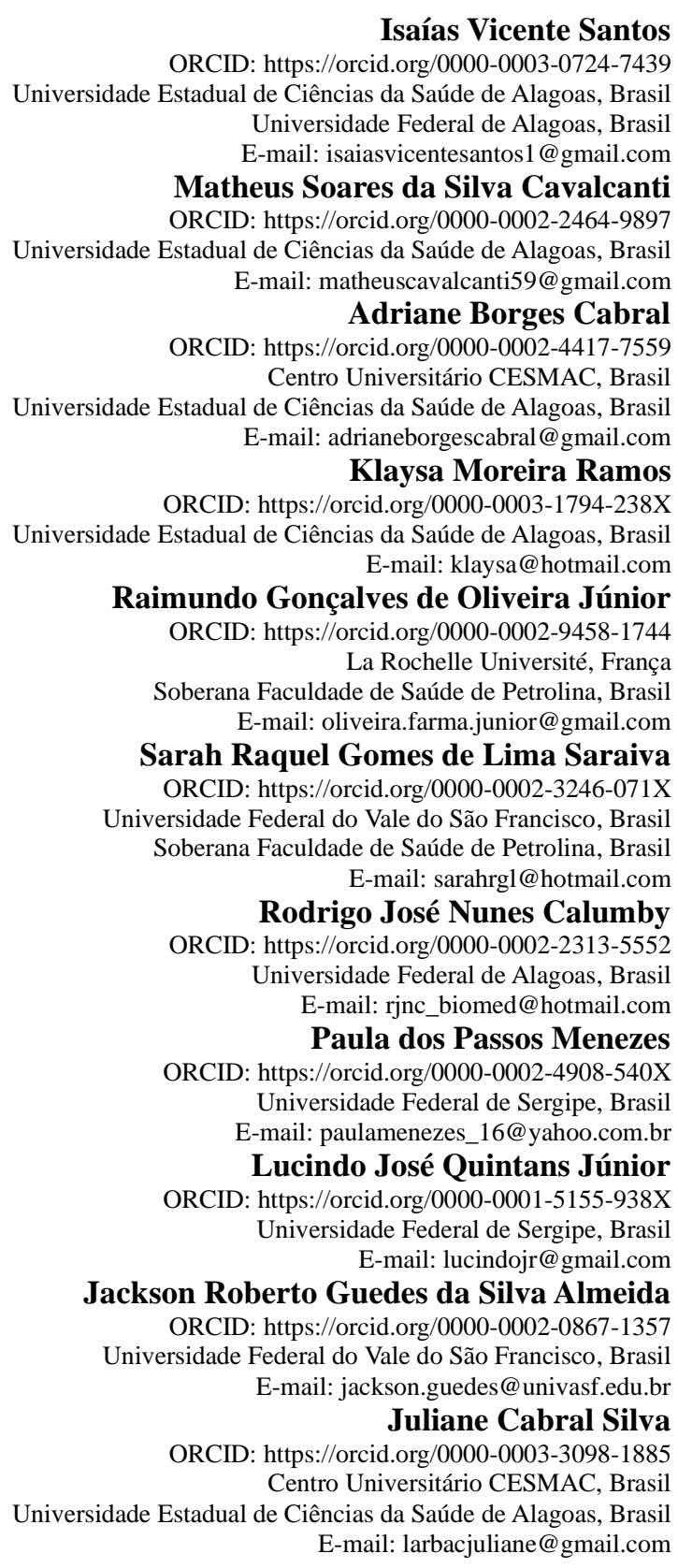

\section{Resumo}

O presente trabalho foi concebido com o objetivo de caracterizar os atuais estudos acerca do uso de óleos essenciais no tratamento de neuralgias em modelo animal. Trata-se de uma revisão sistemática da literatura, onde o levantamento bibliográfico foi realizado no mês de dezembro de 2019, através de pesquisa por via eletrônica, consultando-se as bases de dados Medline-PubMed, LILACS, Scopus e Web of Science. O presente estudo identificou 338 artigos. Foram removidos 324, sendo selecionados 14 artigos após os critérios de inclusão e exclusão estabelecidos. Todos os artigos selecionados foram na língua inglesa, dentre os quais 14 foram realizados no Brasil com pesquisas com 
extratos, óleos essenciais isolados ou complexados. Esta revisão sistemática sugere que os compostos naturais encontrados possuem potenciais terapêuticos para o tratamento de condições de neuralgias.

Palavras-chave: Fitoterapia; Plantas medicinais; Etnofarmacologia.

\begin{abstract}
The present work was designed with the objective of characterizing the current studies on the use of essential oils in the treatment of neuralgia in an animal model. It is a systematic review of the literature, where the bibliotegraphic survey was carried out in December 2019, through electronic research, consulting the Medline-PubMed, LILACS, Scopus and Web of Science databases. The present study identified 338 articles. 324 were removed, and 14 articles were selected after the established inclusion and exclusion criteria. All articles selected were in the English language, among them 14 were carried out in Brazil with research using extracts, isolated or complexed essential oils. This systematic review suggests that the natural compounds found have therapeutic potentials for the treatment of neuralgia conditions.
\end{abstract}

Keywords: Phytotherapy; Medicinal plants; Ethnopharmacology.

\title{
Resumen
}

El presente trabajo fue concebido con el objetivo de caracterizar los estudios actuales sobre el uso de aceites esenciales en el tratamiento de las neuralgias en un modelo animal. Se trata de una revisión sistemática de la literatura, donde se realizó el relevamiento bibliográfico en diciembre de 2019, mediante investigación electrónica, consultando las bases de datos Medline-PubMed, LILACS, Scopus y Web of Science. El presente estudio identificó 338 artículos. Se eliminaron 324 y se seleccionaron 14 artículos después de los criterios de inclusión y exclusión establecidos. Todos los artículos seleccionados fueron en idioma inglés, entre ellos 14 fueron realizados en Brasil con investigaciones utilizando extractos, aceites esenciales aislados o complejados. Esta revisión sistemática sugiere que los compuestos naturales encontrados tienen potencial terapéutico para el tratamiento de las condiciones de neuralgia.

Palabras clave: Fitoterapia; Plantas medicinales; Etnofarmacología.

\section{Introdução}

A dor é um evento comum em diversos cenários que normalmente envolve a assistência à saúde, desde que é o motivo mais frequente de busca de serviço de saúde em pessoas de todas as idades e em diferentes locais do mundo (Dellaroza et al., 2013). A dor é um fenômeno complexo que envolve vertentes físicas, psíquicas, sociais e comportamentais. Por esta razão, é definida como experiência sensorial e emocional desagradável que ocorre através das ativações de receptores e/ou via dolorosa, que pode estar relacionada a lesões teciduais reais ou potenciais e sua percepção é diferenciada de um indivíduo para o outro (Schestatsky, 2008).

Mudanças estruturais ou funcionais em um ou mais nervos levam a uma condição de dor chamada neuralgia, caracterizada e descrita como latejante, parestesia ou choque elétrico, de curta duração e paroxística, variando desde alguns segundos até minutos, com potencial de severidade e frequência bastante mutáveis (Fan et al., 2012).

O tratamento de neuralgias ainda é um grande desafio para os profissionais da área de saúde e para os usuários portadores deste tipo de patologia. Os principais agentes utilizados para o alivio dos sintomas de dor relacionadas a neuralgia são das classes dos opioides, anticonvulsivantes, antidepressivos e anti-inflamatórios, entretanto esses agentes farmacológicos apresentam vários eventos adversos relacionados ao uso de medicamentos que limitam a terapêutica e produzem menor adesão terapêutica (Krzyzanowska \& Avendaño, 2012).

Para minimizar estas limitações do tratamento farmacológico atual, uma abordagem promissora tem sido o desenvolvimento de novas entidades químicas, através de síntese, semi-síntese ou pela obtenção de origem naturail, como plantas medicinais e/ou seus metabólitos secundários ativos. Eles possuem alta eficácia no tratamento de dor, custo relativamente mais baixo e menor incidência de reações adversas, em comparação com as drogas sintéticas (Siqueira-Lima et al., 2016).

Dentre os produtos naturais com potencial terapêutico temos os óleos essenciais (OEs), que são compostos por metabólitos secundários e possuem uma mistura complexa de substâncias voláteis lipofílicas, geralmente odoríferas e líquidas, extraídos de diversas partes das plantas, normalmente medicinais. Tais compostos são constituídos, principalmente, por 
derivados terpênicos como os monoterpenos, diterpenos e sesquiterpenos. Os OEs são utilizados em diversas atividades terapêuticas, in natura ou em preparações farmacêuticas, como efeitos calmantes, anticonvulsivantes, antimicrobiano, fungicida, antioxidante e analgésicos (Eler \& Jaques, 2006; Oussalah et al., 2007; Siqueira Lima et al., 2016).

Várias propostas terapêuticas tem sido propostas neste sentido, a exemplo do medicamento Ziclague ${ }^{\circledR}$ desenvolvido pelo Laboratório Farmacêutico do Grupo Hebron a base de OEs de Alpinia speciosa. Esta é uma planta medicinal amplamente encontrada no nordeste Brasileiro e conhecida popularmente como "colônia" ou "bastão do imperador", sendo o medicamento usado para o manejo da paralisia cerebral, dor e espasticidade (Santos Júnior et al., 2017; Oliveira et al., 2018). Levantamento recente sobre novas patentes do setor farmacêutico com uso de produtos naturais a base de OEs ou seus compostos majoritários tem demonstrado um interesse do setor no tratamento de neuralgias, bem como das "dores disfuncionais" como fibromialgia e dor neuropática (Guimarães et al., 2014; Oliveira et al., 2017; Pina et al., 2017).

Neste contexto, a presente revisão buscou sumarizar a importância dos OEs no tratamento de neuralgias, bem como elucidar suas limitações, através de uma revisão sistemática de estudos recentes que empregam tais produtos naturais para essa afecção dolorosa em modelos animais.

\section{Metodologia}

O presente estudo caracteriza-se por ser uma revisão sistemática da literatura, cujo método permite a inclusão de diversas literaturas científicas, tendo como uma das principais vantagens a possibilidade de combinar sistematicamente as informações de um conjunto de estudos realizados, podendo trazer resultados conflitantes ou até mesmo coincidentes (De-LaTorre-Ugarte-Guanilo, 2011).

A pergunta norteadora para a elaboração da presente revisão sistemática foi: Quais são os OEs utilizados no tratamento de neuralgias em modelos animais? Portanto, utilizamos uma abordagem que analisa a tendência de novas propostas terapêuticas e a consolidação de terapias usadas atualmente.

O levantamento bibliográfico foi realizado no mês de dezembro de 2019, através de pesquisa por via eletrônica, consultando-se os bancos de dados National Library of Medicine, Washington, D.C. (Medline-PubMed), Literatura LatinoAmericana em Ciências da Saúde (LILACS), Scopus e Web of Science.

A busca e seleção dos estudos foram executadas por quatro pesquisadores de forma independente, sendo um quinto pesquisador o juiz. Foram utilizados descritores padronizados pelo Medical Subject Headings (MeSH), cruzando, no formulário básico no PubMed, LILACS, Scopus e Web of Science. Portanto, as combinações utilizadas para busca nas respectivas bases de dados foram: Essential oils AND Neuralgia; Essential oil AND Neuralgia; Terpenes AND Neuralgia.

As estratégias de investigações utilizadas nas respectivas bases de dados e os motivos de exclusão foram apresentadas no fluxograma (Figura 1), seguindo as recomendações do grupo PRISMA (Moher et al., 2015).

Para a inclusão e análise dos artigos, foram estabelecidos os seguintes critérios: artigos indexados nas bases de dados descritas previamente; publicados na íntegra na língua inglesa, espanhola ou portuguesa e artigos originais. Excluiu-se desse estudo artigos repetidos, artigos de revisão e artigos que não envolvessem modelos animais. A exclusão foi realizada pelo título, resumo e leitura do artigo na íntegra. 
Figura 1. Fluxograma, segundo PRISMA, para seleção dos estudos encontrados, Maceió - AL, 2020.

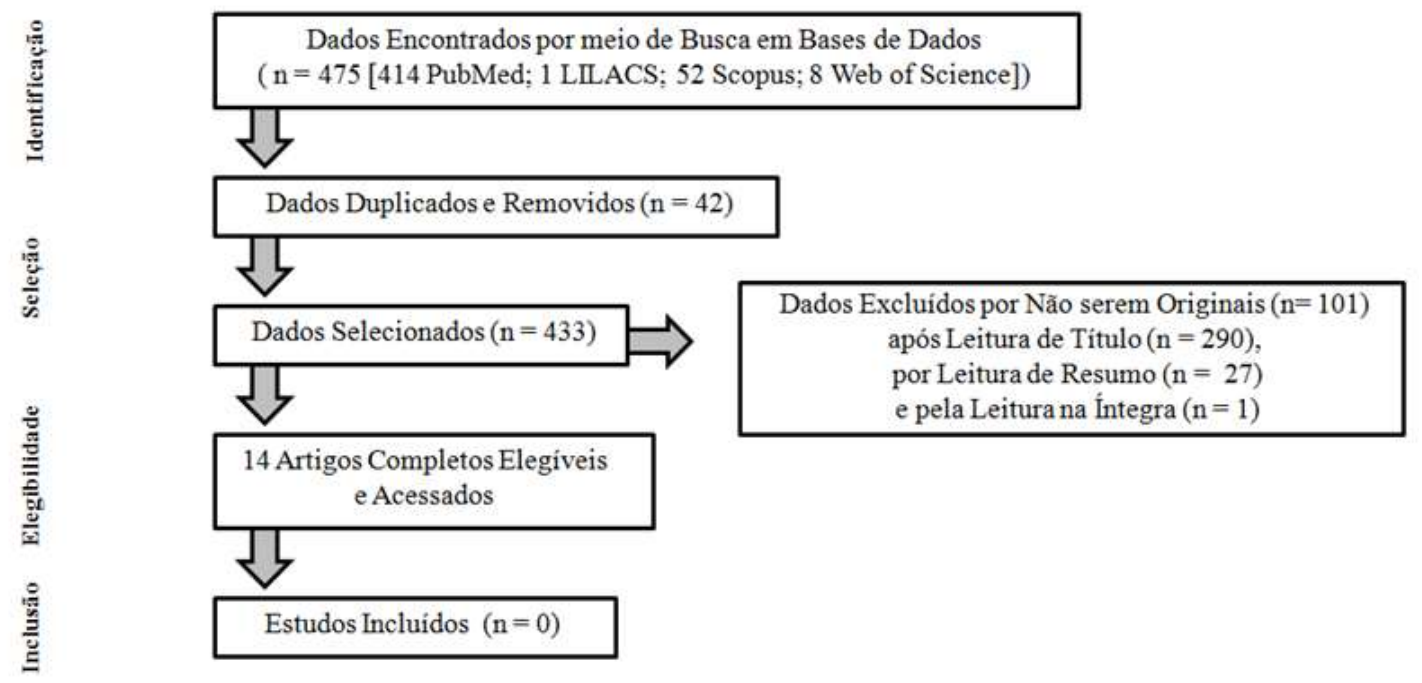

Fonte: Autores.

Para a coleta dos dados, em uma planilha do Excel $^{\circledR}$, foram registrados os dados referentes a cada artigo selecionado (nome, ano, país, substância utilizada, tipo de animal, dose da substância utilizada, parâmetros avaliados quanto ao comportamento do animal e métodos utilizados).

\section{Resultados e Discussão}

O presente estudo identificou no levantamento 475 artigos (414 no PubMed, 1 no LILACS, 52 no Scopus e 8 no Web of Science). Destes, foram removidos 461 artigos (42 artigos por repetição, 101 por não serem originais, 290 pelo título, 27 pelo resumo e 1 pela leitura na íntegra), sendo selecionados 14 artigos após os critérios de inclusão e exclusão estabelecidos.

Curiosamente, dos 14 estudos finais selecionados, a maior parte foi realizado no Brasil (57.14\%), 28.57\% na China e $14.29 \%$ no Irã (Tabela 1). Todavia, $100 \%$ dos estudos selecionados encontram-se publicados na íntegra na língua inglesa. Um dos fatores que pode contribuir para este achado é que o Brasil possui cerca de $20 \%$ da biodiversidade mundial, que tem sido uma fonte de exploração (de maneira geral não sustentável) e estudos, mas especialmente de interesse de grandes empresas multinacionais dos setores farmacêuticos e de biotecnologia para o desenvolvimento de novos fármacos (Lawson, 2009; Oliveira et al., 2012). Quanto aos métodos utilizados pelos autores para avaliação da neuralgia em modelos animais, observouse que o mais frequente foi o Teste de von Frey, que avalia a hipersensibilidade mecânica através do parâmetro de retirada da pata do animal, "flich", entre outros comportamentos (Chaplan et al., 1994).

Já com relação ao tratamento, é notório que as plantas são utilizadas para fins terapêuticos, inclusive dentro das políticas públicas de saúde, e contribuem para a obtenção de diversos fármacos utilizados na clínica médica. Uma prova disso é que cerca de quase a metade dos medicamentos no mundo atualmente derivam, direta ou indiretamente, de fontes naturais (Kumari et al., 2012). Especificamente no manejo da neuralgia, dentre os artigos inseridos no estudo, aproximadamente 43\% utilizaram extratos de plantas medicinais contendo diversos metabólitos secundários e 57\% usaram óleos essenciais de plantas. Os extratos de plantas consistem em uma mistura de diversos componentes químicos que são inativos ou parcialmente ativos e agem em diferentes receptores farmacológicos; os óleos essenciais, por sua vez, concentram especialmente terpenos (Ferreira \& Pinto, 2010). 
Tabela 1. Descrição das características dos estudos inseridos na revisão, Maceió - AL, 2020.

\begin{tabular}{|c|c|c|c|c|c|}
\hline Autor, ano, país & Produto Natural & Animais (sexo) & Dose & Parâmetros avaliados & Métodos utilizados \\
\hline $\begin{array}{c}\text { Quintão et al., 2010, } \\
\text { Brasil }\end{array}$ & Ugni myricoides & $\begin{array}{l}\text { Camundongos } \\
\text { (fêmeas) }\end{array}$ & $\begin{array}{c}5-50 \\
\mathrm{mg} / \mathrm{kg} \\
\text { (v.o.) }\end{array}$ & $\begin{array}{c}\text { Avaliar a sensibilidade } \\
\text { tecidual ao estímulo } \\
\text { mecânico. }\end{array}$ & Teste de von Frey. \\
\hline $\begin{array}{l}\text { Batista et al., 2010, } \\
\text { Brasil }\end{array}$ & ( - ) - linalol & $\begin{array}{l}\text { Camundongos } \\
\text { (fêmeas) }\end{array}$ & $\begin{array}{l}50-200 \\
\mathrm{mg} / \mathrm{kg} \\
\text { (i.p.) }\end{array}$ & $\begin{array}{c}\text { Avaliar a sensibilidade } \\
\text { tecidual ao estímulo } \\
\text { mecânico. }\end{array}$ & Teste de von Frey. \\
\hline $\begin{array}{l}\text { Silva et al., 2011, } \\
\text { Brasil }\end{array}$ & $\alpha-\beta-$ amirina & Ratos (machos) & $\begin{array}{c}30 \\
\mathrm{mg} / \mathrm{kg} \\
\text { (v.o.) }\end{array}$ & $\begin{array}{l}\text { Avaliar a sensibilidade } \\
\text { tecidual ao estímulo } \\
\text { mecânico, desempenho } \\
\text { motor, retirada da cauda. }\end{array}$ & $\begin{array}{l}\text { Teste de von Frey, } \\
\text { Teste de Rota-Rod, } \\
\text { Teste de tail-flick. }\end{array}$ \\
\hline Liu et al., 2012, China & $\begin{array}{l}\text { Glicosídeo iridóide } \\
\text { [P. scandens } \\
\text { (LOUR.) MERRILL } \\
\text { (Rubiaceae)] }\end{array}$ & Ratos (machos) & $\begin{array}{l}70-280 \\
\mathrm{mg} / \mathrm{kg} \\
\text { (i.p.) }\end{array}$ & $\begin{array}{c}\text { Avaliar a sensibilidade } \\
\text { tecidual ao estímulo } \\
\text { mecânico. }\end{array}$ & Teste de von Frey. \\
\hline $\begin{array}{c}\text { Dutra et al., 2012, } \\
\text { Brasil }\end{array}$ & Eufol & $\begin{array}{l}\text { Camundongos } \\
\text { (machos) }\end{array}$ & $\begin{array}{l}3-100 \\
\mathrm{mg} / \mathrm{kg} \\
\text { (v.o.) }\end{array}$ & $\begin{array}{c}\text { Avaliar a sensibilidade } \\
\text { tecidual ao estímulo } \\
\text { mecânico. }\end{array}$ & Teste de von Frey. \\
\hline $\begin{array}{l}\text { Amin; Hosseinzadeh, } \\
\text { 2012, Irã }\end{array}$ & $\begin{array}{l}\text { Extrato de Açafrão } \\
\text { (Crocus sativus L.) }\end{array}$ & Ratos (machos) & $\begin{array}{l}50-200 \\
\mathrm{mg} / \mathrm{kg} \\
\text { (i.p.) }\end{array}$ & $\begin{array}{l}\text { Avaliar a sensibilidade } \\
\text { tecidual ao estímulo } \\
\text { mecânico, estímulo } \\
\text { térmico. }\end{array}$ & $\begin{array}{c}\text { Teste de von Frey, } \\
\text { Teste de Placa } \\
\text { Quente. }\end{array}$ \\
\hline $\begin{array}{l}\text { Wang et al., 2014, } \\
\text { China }\end{array}$ & Catalpol & Ratos (machos) & $\begin{array}{l}1-125 \\
\mathrm{mg} / \mathrm{kg} \\
\text { (i.p) }\end{array}$ & $\begin{array}{c}\text { Avaliar a sensibilidade } \\
\text { tecidual ao estímulo } \\
\text { mecânico. }\end{array}$ & Teste de von Frey. \\
\hline $\begin{array}{c}\text { Piccinelli et al., 2014, } \\
\text { Brasil }\end{array}$ & $\begin{array}{l}\text { Schinus } \\
\text { terebinthifolius }\end{array}$ & Ratos (machos) & $\begin{array}{l}3-100 \\
\mathrm{mg} / \mathrm{kg} \\
\text { (v.o.) }\end{array}$ & $\begin{array}{l}\text { Avaliar a sensibilidade } \\
\text { tecidual ao estímulo } \\
\text { mecânico, ansiedade }\end{array}$ & $\begin{array}{l}\text { Teste de von Frey, } \\
\text { Teste de Campo } \\
\text { Aberto. }\end{array}$ \\
\hline $\begin{array}{l}\text { Yang et al., 2014, } \\
\text { China }\end{array}$ & Celastrol & Ratos (machos) & $\begin{array}{c}0,3 \\
\mathrm{mg} / \mathrm{kg} \\
\text { (i.p) }\end{array}$ & $\begin{array}{c}\text { Avaliar a sensibilidade } \\
\text { tecidual ao estímulo } \\
\text { mecânico. }\end{array}$ & Teste de von Frey. \\
\hline $\begin{array}{c}\text { Nishijima et al., 2014, } \\
\text { Brasil }\end{array}$ & Citral & $\begin{array}{l}\text { Camundongos } \\
\text { (machos) }\end{array}$ & $\begin{array}{l}25-300 \\
\mathrm{mg} / \mathrm{kg} \\
\text { (i.g.) }\end{array}$ & $\begin{array}{l}\text { Desempenho motor, } \\
\text { ansiedade. }\end{array}$ & $\begin{array}{c}\text { Teste de Rota-Rod, } \\
\text { Teste de Campo } \\
\text { aberto. }\end{array}$ \\
\hline $\begin{array}{c}\text { Jiang et al., 2015, } \\
\text { China }\end{array}$ & $(+)$ - Borneol & Ratos (machos) & $\begin{array}{c}125- \\
500 \\
\mathrm{mg} / \mathrm{kg} \\
\text { (v.o.) }\end{array}$ & $\begin{array}{l}\text { Avaliar a sensibilidade } \\
\text { tecidual ao estímulo } \\
\text { mecânico, Desempenho } \\
\text { motor. }\end{array}$ & $\begin{array}{l}\text { Teste de von Frey, } \\
\text { Teste de Rota-Rod. }\end{array}$ \\
\hline $\begin{array}{l}\text { Nucci-Martins et al., } \\
\text { 2015, Brasil }\end{array}$ & Pterodon pubescens & $\begin{array}{l}\text { Camundongos } \\
\text { (machos) }\end{array}$ & $\begin{array}{c}30-300 \\
\mathrm{mg} / \mathrm{kg} \\
\text { (v.o.) }\end{array}$ & $\begin{array}{l}\text { Avaliar a sensibilidade } \\
\text { tecidual ao estímulo } \\
\text { mecânico, estímulo } \\
\text { térmico, Ansiedade, } \\
\text { atividade locomotor. }\end{array}$ & $\begin{array}{l}\text { Teste de von Frey, } \\
\text { Teste de Placa } \\
\text { Quente, Teste de } \\
\text { Campo Aberto. }\end{array}$ \\
\hline $\begin{array}{l}\text { Khalilzadeh et al., } \\
\text { 2015, Irã }\end{array}$ & Vitex agnus-castus & Ratos (machos) & $\begin{array}{c}100- \\
200 \\
\mathrm{mg} / \mathrm{kg} \\
\text { (s.c.) }\end{array}$ & $\begin{array}{l}\text { Friccionar os olhos com } \\
\text { a pata traseira. }\end{array}$ & $\begin{array}{l}\text { Teste do olho (teste } \\
\text { trigeminal agudo). }\end{array}$ \\
\hline $\begin{array}{c}\text { Paula-Freire et al., } \\
\text { 2016, Brasil }\end{array}$ & $\begin{array}{l}\text { Ocimum } \\
\text { gratissimum }\end{array}$ & $\begin{array}{l}\text { Camundongos } \\
\text { (macho) }\end{array}$ & $\begin{array}{l}20-40 \\
\mathrm{mg} / \mathrm{kg} \\
\text { (v.o.) }\end{array}$ & $\begin{array}{c}\text { Avaliar a sensibilidade } \\
\text { tecidual ao estímulo } \\
\text { mecânico, estímulo } \\
\text { térmico. }\end{array}$ & $\begin{array}{c}\text { Teste de von Frey, } \\
\text { Teste de Placa } \\
\text { Quente. }\end{array}$ \\
\hline
\end{tabular}


Para que haja uma melhor compreensão dos resultados, resumimos os produtos naturais utilizados nos estudos em substâncias isoladas, espécies de plantas, óleos essenciais e extratos de plantas medicinais contendo metabólitos secundários, que são descritos a seguir.

\subsection{Substâncias Isoladas}

Dentre as substâncias isoladas encontradas nos artigos avaliados na presente revisão sistemática, todos foram derivados da classe dos terpenóides, também conhecidos como isoprenóides. As moléculas pertencentes à essa classe de produtos naturais, destacam-se pela presença de unidades de repetição ramificadas de cinco carbonos, semelhantes às unidades de isopreno $\left(\mathrm{C}_{5} \mathrm{H}_{8}\right)$. Deste modo, a nomenclatura dos terpenóides depende do número de estruturas de isopreno e pode ser classificada como monoterpeno $\left(\mathrm{C}_{10} \mathrm{H}_{\mathrm{x}} \mathrm{O}_{\mathrm{y}}\right)$, sesquiterpeno $\left(\mathrm{C}_{15} \mathrm{H}_{\mathrm{x}} \mathrm{O}_{\mathrm{y}}\right)$, diterpeno $\left(\mathrm{C}_{20} \mathrm{H}_{\mathrm{x}} \mathrm{O}_{\mathrm{y}}\right)$, triterpeno $\left(\mathrm{C}_{30} \mathrm{H}_{\mathrm{x}} \mathrm{O}_{\mathrm{y}}\right)$, tetraterpeno $\left(\mathrm{C}_{40} \mathrm{H}_{\mathrm{x}} \mathrm{O}_{\mathrm{y}}\right)$ e politerpeno $\left(\mathrm{C}_{>40} \mathrm{H}_{\mathrm{x}} \mathrm{O}_{\mathrm{y}}\right)$ (Aldred et al., 2009). No âmbito desta revisão, os artigos relataram o uso de monoterpenos e triterpenos nos estudos de neuralgia utilizando modelo animal.

\subsubsection{Monoterpenos}

Os monoterpenos $\left(\mathrm{C}_{10} \mathrm{H}_{\mathrm{x}} \mathrm{O}_{\mathrm{y}}\right)$ são dímeros de isopreno e podem ser divididos em compostos acíclicos, monocíclicos, bicíclicos e tricíclicos. Os monoterpenos e seus derivados, como é o caso dos iridóides (monoterpeno + glicose), são de suma importância nas indústrias farmacêutica e cosmética, bem como na produção de aditivos e pesticidas aromatizantes. Todavia, sua estrutura única (Figura 2) é frequentemente associada com alta volatilidade, característica que reduz a biodisponibilidade dos fármacos derivados desta classe. Em função disso, estudos recentes têm destacado o uso de carreadores como ciclodextrinas, micropartículas e nanopartículas, como estratégias farmacotécnicas de carrear o fármaco até o local de ação, melhorando a sua biodisponibilidade (Lima et al., 2016).

Figura 2. Monoterpenos de maior ocorrência nos artigos avaliados na presente revisão. Desenhado no software ChemDraw Ultra, Maceió - AL, 2020.
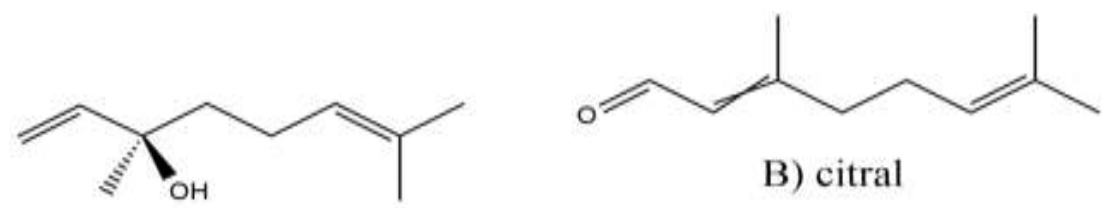

B) citral

A) (-)-linalol

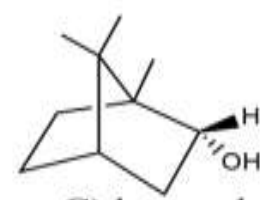

C) borneol

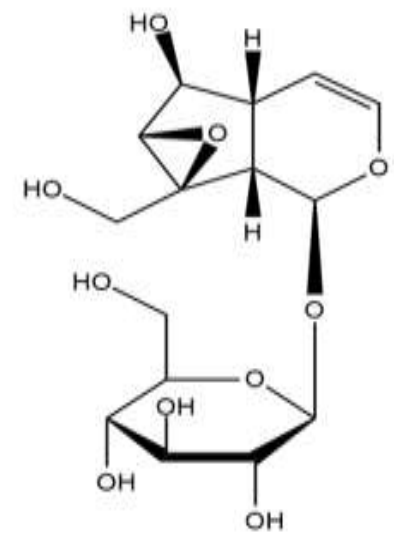

D) catalpol

Fonte: Autores. 


\subsubsection{1 (-)-Linalol}

O (-)-linalol (Figura 2A) é um monoterpeno, composto predominante em OEs de espécies de plantas aromáticas, como por exemplo em OEs de Ocimum basilicum ("manjericão") e Ocimum gratissimum ("alfavacão") (Elisabetsky et al., 1999). Diversos estudos mostraram efeitos desse monoterpeno. O uso isolado de (-)-linalol apresentou efeitos antinociceptivos significativos em camundongos, bem como reduziu a hipersensibilidade mecânica e a inflamação causada por injeção intraplantar de adjuvante completo de Freund (Batista et al., 2010). É evidenciado que o (-)-linalol e o OE de Ocimum gratissimum apresentaram efeitos anti-hiperalgésico em modelos experimentais de fibromialgia por modular área centrais relacionadas com as vias de transmissão da dor, com possível envolvimento dos sistemas opioides e serotoninérgico (Nascimento et al., 2014). Os efeitos analgésicos e anti-inflamatórios do (-)-linalol estão relacionados quanto a sua capacidade de reduzir citocinas inflamatórias tais como TNFa e pela participação dos receptores opioidérgicos (Quintans Júnior et al., 2013).

\subsubsection{Citral}

O citral (Figura 2B) é um monoterpeno volátil e pode ser encontrado nas plantas Cymbopogon citratus e Lippia alba (Sousa et al., 2015). Este monoterpeno vem demonstrando interesse farmacológico visto que já foram comprovados diversos efeitos, como a inibição da liberação de citocinas IL-1 $1 \beta$ e IL-6 e antinociceptivo, quando administrado via intraperitonial (Quintans Júnior et al., 2016). Nishijima et al. (2014) constataram que a administração do citral por gavagem intragástrica inibiu significativamente as fases inflamatórias induzidas pelo teste da formalina no edema de pata, comparado com o grupo controle. Além disso, o citral reduziu a hiperalgesia mecânica, característica clínica comum em pacientes com dor. Adicionalmente ao seu efeito antinociceptivo, os autores verificaram que este monoterpeno exibiu ação gastroprotetora significativa contra úlceras induzidas, demonstrando deste modo o potencial do citral como um fármaco para o tratamento de diversos modelos de neuralgias. O citral também atua sobre os receptores GABAérgicos produzindo inibição da hiperatividade cerebral e podendo atuar como um estabilizador de membrana através dos bloqueio de canais iônicos para $\mathrm{Na}^{+}$dependentes de voltagens (Sousa et al., 2015).

\subsubsection{3 (+)-Borneol}

Estudos recentes relatam que o (+)-borneol (Figura 2C) apresenta diversos efeitos farmacológicos, incluindo antiinflamatórios e neuroprotetor (Liu et al., 2011). Estudos demonstraram que o borneol produziu efeitos analgésicos devido aos seus efeitos inibitórios na liberação ou síntese de mediadores inflamatórios, como IL-1 $\beta$, TNF- $\alpha$ e NK-кB (Almeida et al., 2013). Jiang et al. (2015) demonstraram que o (+)-borneol exibiu efeito antinociceptivo significativo em neuralgias, sem produzir déficit motor. Todavia, um estudo demonstrou que o (+)-borneol apresenta forte efeito analgésico em humanos em formulação tópica através do envolvimento de receptores TRPM8 o qual tem papel promissor no manejo das neuralgias (Wang et al., 2017).

\subsubsection{Catalpol}

Segundo Bi et al. (2013), o catalpol (Figura 2D) é um iridoide glicosilado, composto ativo presente em plantas medicinais importantes, como a Rehmannia glutinosa, que é utilizada no tratamento de diversas patologias na medicina tradicional chinesa. Segundo Wang et al. (2014), seu estudo foi o primeiro a avaliar os efeitos analgésicos do catalpol em neuralgias, fornecendo evidências de que o catalpol proporcionou acentuado alívio em roedores, provavelmente estando associado à inibição da forma fagocitária ativa da micróglia, citocinas (IL-1 $\beta$, IL-6, e TNF- $\alpha$ ) e Fator Nuclear kappa B (NK$\kappa B)$. Estes efeitos parecem estar relacionados com a capacidade do catalpol produzir uma modulação da neuroinflamação na 
medula espinhal pela inibição de citocinas pró-inflamatórias. Mas sua ação sobre a $\beta$-endorfina no hipocampo e em áreas de controle da dor podem igualmente contribuir para o controle da neuralgia (Jia et al., 2015).

\subsubsection{Glicosídeo iridóide [P. scandens (LOUR.) MERRILL (Rubiaceae)]}

O glicosídeo iridoide de P. scandens (GIPS) é composto por paederosida, scandoside e asperulosida, que são componentes ativos principais desta planta. Ma et al. (2009), demonstraram em seus estudos que a administração oral do extrato de $P$. scandens em ratas com artrite gotosa provocou propriedades antinociceptivas e anti-inflamatórias. Segundo Liu et al. (2012), o GIPS mostrou efeito antinociceptivo contra a lesão do nervo periférico em modelo de dor neuropática em ratos, sugerindo desta forma a eficácia como um agente terapêutico analgésico.

\subsubsection{Triterpenos}

Triterpenos (Figura 3) são alcenos naturais oriundos de vegetais, animais ou fungos. Sua estrutura inclui 30 elementos de carbono, sendo, portanto, constituídos por seis unidades isoprênicas. Quanto à estrutura, os triterpenos podem ser classificados em tetracíclico e pentacíclico. Esta classe de produtos naturais possui diversos usos além do seu potencial terapêutico, tais como, flavorizantes de alimentos, pigmentos, polímeros, fibras, colas e ceras (Parmar et al., 2013; Chudzik et al., 2015).

Figura 3. Triterpenos mais relatados nos artigos avaliados na presente revisão. Desenhado no software ChemDraw Ultra, Maceió - AL, 2020.

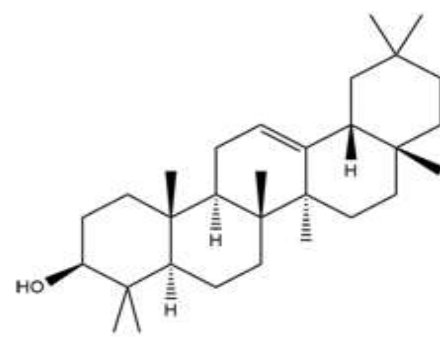

A) amirina<smiles>CC(C)[C@H]1CC[C@]2(C)C3=C(CC[C@]12C)[C@@]1(C)CC[C@H](O)C(C)(C)[C@@]31C</smiles>

B) eufol<smiles>CC1=C(O)C(=O)C=C2C1=CC=C1[C@@]3(C)CC[C@]4(C)CCC(C)(C)C[C@]4(C)[C@]3(C)CC[C@@]21C</smiles>

C) celastrol

Fonte: Autores. 


\subsubsection{Amirina}

A $\alpha-\beta$-amirina (Figura 3A) é um triterpeno pentacíclico e seus efeitos anti-inflamatórios e antinociceptivos têm sido evidenciados na literatura, quando a substância é administrada em modelos de nocicepção aguda. A amirina pode ser encontrada nas plantas Protium kleinii e Protium heptaphyllum (Otuki et al., 2005). Foi evidenciado que este triterpeno evitou a produção de mediadores inflamatórios responsáveis pelo progresso da inflamação induzida por citocinas (Silva et al., 2011), e ratifica que esse composto pode ter relevância clínica e é uma potente substância de interesse para alívio de neuralgias. Adicionalmente, é demonstrado que a $\alpha$ - $\beta$-amirina atenua a dor orofacial através do envolvimento do sistema opioide periférico e dos receptores TRP (Transient receptor potential channel), alvos importantes para o controle das neuralgias (Holanda Pinto et al., 2008).

\subsubsection{Eufol}

Estudos mostraram que o eufol (Figura 3B) inibiu significativamente a hiperalgesia mecânica causada por mediadores inflamatórios (IL-1 $\beta$, IL-6, CXCL1/KC e TNF- $\alpha$ ). Adicionalmente, o quimiotipo encontrado pela primeira vez na seiva de Euphorbia tirucalli exibiu propriedades anti-inflamatórias e antinociceptivas quando administradas por via oral em camundongos. É evidenciado também que este triterpeno tetracíclico atua através da ativação de receptores canabinóides (CB1 e CB2), portanto, produzindo analgesia bem como inibindo citocinas inflamatórias importantes na indução das neuralgias (Dutra et al., 2012).

\subsubsection{Celastrol}

O celastrol (Figura 3C) é um triterpeno pentacíclico, utilizado cotidianamente na medicina chinesa. Relatos apontam que esta molécula exerce grande efeito anti-inflamatório sobre a doença de Alzheimer, lúpus eritematoso sistêmico, artrite reumatoide, asma e aterosclerose (Paris et al., 2010; Venkatesha et al., 2011; Nanjundaiah et al., 2012). Yang et al. (2014) verificaram que após indução da neuralgia através de injeção de carragenina na pata esquerda dos ratos e avaliação do comportamento da dor, através do teste de von Frey em resposta à administração intraperitoneal do triterpeno, o celastrol demonstrou além da ação anti-inflamatória, efeito antinociceptivo sobre a neuralgia induzida. Adicionalmente, o celastrol reduziu o comportamento doloroso em roedores no modelo animal de osteoartrite através da supressão da via da quimiocina de açãopleiotrópica, com expressão alterada em tecidos inflamados e lesionados - SDF-1/CXCR4 (stromal cell-derived factor 1/receptor C-X-C chemokine receptor type 4) (Wang et al., 2018).

\subsection{Espécies de plantas, óleos essenciais e extratos de plantas medicinais contendo metabólitos secundários}

\subsubsection{Ugni myricoides}

Estudo demonstra efeitos anti-inflamatórios do extrato de Ugni myricoides em modelo de edema de pata induzido por carragenina em ratos (Orhan et al., 2006). Foi notado que o extrato de Ugni myricoides foi capaz de retardar o aparecimento de resposta hipernociceptiva mecânica e redução significativa da sensibilidade mecânica até 48 horas após administração oral do extrato em camundongos (Quintão et al., 2010). Quintão et al. (2010) apresentaram provas convincentes que os efeitos citados anteriormente estão relacionados com a presença do principal composto do extrato de Ugni myricoides, o monoterpeno $\alpha$ pineno.

\subsubsection{Extrato de Açafrão (Crocus sativus L.)}

O açafrão é um extrato da Crocus sativus L. que tem como os principais constituintes bioativos a picrocrocina (percursor do safranal) e acrocina. Estudos in vivo e in vitro demonstraram que o açafrão tem ação antioxidante, 
anticonvulsivante e antidepressiva (Hosseinzadeh et al., 2004; Hosseinzadeh \& Talebzadeh, 2005; Hosseinzadeh et al., 2009). Outros trabalhos reportaram efeitos antinociceptivos e anti-inflamatórios de extrato aquoso de açafrão em edema induzido por xilol, teste da placa quente e teste de formalina em modelo animal (Hosseinzadeh \& Younesi, 2002). Amin \& Hosseinzadeh (2002) avaliaram a eficácia da administração sistêmica de extratos aquosos de açafrão e de seus principais constituintes bioativos (safranal e crocina) em modelo crônico de neuralgia em ratos, mimetizando os sintomas de compressão crônica do nervo ciático em humanos. Para tal, os extratos foram administrados durante 7 dias consecutivos, por via intraperitoneal, e observou-se que os princípios ativos atenuaram a alodinia mecânica e a hiperalgesia térmica. Deste modo foi demonstrado que o extrato aquoso de açafrão e os seus constituintes são úteis no tratamento de neuralgia.

\subsubsection{Schinus terebinthifolius}

$\mathrm{Na}$ medicina popular, as cascas, folhas e frutos do Schinus terebinthifolius possuem uma alta concentração de monoterpenos, principalmente limoneno e $\alpha$-felandreno. Por essa razão, o Schinus terebinthifolius vem sendo utilizado como agente anti-inflamatório, cicatrizante e analgésico (Santos et al., 2012). Lappas \& Lappas (2012) demonstraram que o monoterpeno limoneno tem papel imunossupressor, sendo capaz de inibir efeitos de citocinas inflamatórias em linfócitos T. Estudos realizados por Lima et al. (2012) mostraram tanto efeitos anti-hiperalgésicos como anti-inflamatórios do $\alpha$-felandreno em roedores, por um mecanismo relacionado com opioides. Piccinelli et al. (2015), por sua vez, evidenciaram em seus resultados, no teste em campo aberto, que o óleo essencial ou os compostos administrados via oral, durante 15 dias, não diminuíram a atividade locomotora comparada com o grupo controle, e demonstraram também a atividade anti-hiperalgésica dos dois monoterpenos encontrados na planta Schinus terebinthifolius: limoneno e $\alpha$-felandreno.

\subsubsection{Pterodon pubescens}

Conhecida como "sucupira-branca", é uma árvore nativa do Brasil que vem sendo usada na medicina popular pela sua ação anti-inflamatória e analgésica (Carvalho, 2004). Em um estudo anterior foi demonstrado que, após a administração oral, o extrato de Pterodon pubescens provocou inibição significativa nas fases do teste de formalina, hiperalgesia mecânica e térmica na dor pós-operatória em roedores (Nucci et al., 2012). Nucci-Martins et al. (2015) demonstraram em seus estudos o efeito analgésico do extrato de Pterodon pubescens em neuralgia, mostrando que o tratamento oral com esse extrato proporcionou inibição da hiperalgesia mecânica e térmica induzida no nervo ciático em ratos. Além disso, os autores relataram que após a administração oral prolongada de $300 \mathrm{mg} / \mathrm{kg}$ do extrato durante 10 dias, não foi apresentado efeito de toxicidade. Deste modo, evidências científicas vêm acrescentando e destacando o potencial da planta medicinal Pterodon pubescens no desenvolvimento de fito medicamentos com propriedades analgésicas.

\subsubsection{Vitex agnus-castus}

O Vitex agnus-castus (VAC) é uma pequena planta distribuída amplamente no Oriente Médio e Mediterrâneo (Stojkovic et al., 2011). A VAC é empregada, tradicionalmente, para tratamento de problemas menstruais, inflamação, disfunção sexual, ansiedade e dor (Upton, 2001). Na medicina popular iraniana, a VAC é utilizada para tratamento de epilepsia, constipação, edema e sedação e os óleos essenciais extraídos dessa planta possuem propriedades anti-inflamatórias e efeitos antinociceptivos em neuralgia, além disso o VAC apresenta atividades antimicrobianas e antifúngicas (Saberi et al., 2008, Choudhary et al., 2009). Khalilzadeh et al. (2015) realizaram o teste trigeminal agudo, aplicando uma gota de cloreto de sódio na superfície da córnea de ratos para avaliar a quantidade de vezes que os animais limpavam os olhos com a pata dianteira ou com a pata traseira. Após administração subcutânea de óleos essenciais presentes no extrato da VAC nas doses de 100,150 e $200 \mathrm{mg} / \mathrm{kg}$, foi evidenciado efeito inibitório na resposta à limpeza dos olhos, porém o efeito antinociceptivo desses 
óleos essenciais foram inibidos após administração de naloxona $(1 \mathrm{mg} / \mathrm{kg})$ e atropina $(1 \mathrm{mg} / \mathrm{kg})$ via intraperitoneal. Deste modo, os autores ratificam que os óleos essenciais presentes no extrato da VAC produziram atividade analgésica neste modelo.

\subsubsection{Ocimum gratissimum}

O Ocimum gratissimum é uma planta encontrada amplamente no Brasil, sendo utilizada na cozinha como o condimento conhecido como "manjericão". No estado da Amazônia, o extrato dessa planta é utilizado para cicatrização de feridas. O óleo essencial de $O$. gratissimum contém os constituintes químicos eugenol e mirceno, que são monoterpenos com atividade antinociceptiva (Paula-Freire et al., 2013). Testes realizados para tratamento com o extrato da planta $O$. gratissimum contendo eugenol e mirceno em neuralgia induzida por lesão do nervo ciático em ratos, apresentou uma acentuada hipernocicepção térmica e mecânica (Dworkin et al., 2003). Paula-Freire et al. (2016) demonstraram que o extrato aquoso do O. gratissimum e seus componentes ativos testados mostraram atividades antinociceptivas em modelo de dor induzida por lesão do nervo ciático em camundongos após a administração oral desse extrato $(20-40 \mathrm{mg} / \mathrm{kg})$, contribuindo para ampliar as atividades biológicas desta planta e assim justificar o seu uso popular.

\section{Considerações Finais}

Esta revisão sistemática sugere que os compostos naturais apresentados possuem efeitos antinociceptivos nos modelos de neuralgias, porém todos os estudos encontrados nesta pesquisa encontram-se nas fases iniciais, não estando, ainda, diretamente relacionados com uma possível aplicabilidade clínica.

Dessa forma, estudos farmacocinéticos, farmacodinâmicos e de toxicidade destes fármacos são necessários para que seja oferecida uma possibilidade de desenvolvimento de novos medicamentos para o tratamento mais adequado da dor proveniente de neuralgias. Tendo em vista que os tratamentos farmacológicos comercialmente disponíveis, possuem diversos efeitos adversos significativos nestas condições clínicas.

\section{Agradecimentos}

Agradecemos à Fundação de Amparo à Pesquisa do Estado de Alagoas (FAPEAL).

\section{Referências}

Aldred, E. M., Buck, C., \& Vall, K. (2009). Pharmacology. Churchill Livingstone, 352p.

Almeida, J. R., Souza, G. R., Silva, J. C., Saraiva, S. R., Júnior, R. G., Quintans, J., Barreto, R., Bonjardim, L. R., Cavalcanti, S. C., \& Quintans Júnior, L. J. (2013). Borneol, a bicyclic monoterpene alcohol, reduces nociceptive behavior and inflammatory response in mice. The Scientific World Journal, 2013, 808460 .

Amin, B., \& Hosseinzadeh, H. (2012). Evaluation of aqueous and ethanolic extracts of saffron, Crocus sativus L., and its constituents, safranal and crocin in allodynia and hyperalgesia induced by chronic constriction injury model of neuropathic pain in rats. Fitoterapia, 83(5), 888-895.

Batista, P. A., Werner, M. F. P., Oliveira, E. C., Burgos, L., Pereira, P., Brum, L. F. S., Story, G. M., \& Santos, A. R. S. (2010). The antinociceptive effect of ()-linalool in models of chronic inflammatory and neuropathic hypersensitivity in mice. Journal of Pain, 11(11), $1222-1229$.

Bi, J., Jiang, B., Zorn, A., Zhao, R. G., Liu, P., \& An, L. J. (2013). Catalpol inhibits LPS plus IFN- $\gamma$-induced inflammatory response in astrocytes primary cultures. Toxicology in Vitro, 27(2), 543-550.

Carvalho, J. C. T. (2004). Fitoterápicos anti-inflamatórios: aspectos químicos, farmacológicos e aplicações terapêuticas. Tecmedd. Ribeirão Preto: São Paulo, 480p.

Chaplan, S. R., Bach, F. W., Pogrel, J. W., Chung, J. M., \& Yaksh, T. L. (1994). Quantitative assessment of tactile allodynia in the rat paw. Journal of Neuroscience Methods, 53(1), 55-63.

Choudhary, M. I., Azizuddin, S. J., Nawaz, S. A., Khan, K. M., Tareen, R. B., \& Atta-ur-Rahman. (2009). Antiinflammatory and lipoxygenase inhibitory compounds from Vitex agnus-castus. Phytotherapy Research, 23(9), 1336-1339.

Chudzik, M., Korzonek-Szlacheta, I., \& Król, W. (2015). Triterpenes as potentially cytotoxic compounds. Molecules (Basel, Switzerland), 20(1), 1610-1625. 
De-la-Torre-Ugarte-Guanilo, M. C., Takahashi, R. F., \& Bertolozzi, M. R. (2011). Systematic review: general notions. Revista da Escola de Enfermagem da USP, 45(5), 1260-1266.

Dellaroza, M. S. G., Pimenta, C. A. M., Lebrão, M. L., \& Duarte, Y. A. (2013). Associação de dor crônica com uso de serviços de saúde em idosos residentes em São Paulo. Revista de Saúde Pública, 47(5), 914-922.

Dutra, R. C., Silva, K. A. B. S., Bento, A. F., Marcon, R., Paszcuk, A. F., Meotti, F. C., Pianowski, L. F., \& Calixto, J. B. (2012). Euphol, a tetracyclic triterpene produces antinociceptive effects in inflammatory and neuropathic pain: the involvement of cannabinoid system. Neuropharmacology, 63(4), 593605.

Dworkin, R. H., Backonja, M., Rowbotham, M. C., Allen, R. R., Argoff, C. R., Bennett, G. J. et al. (2003). Advances in neuropathic pain: diagnosis, mechanisms, and treatment recommendations. Archives of Neurology, 60(11), 1524-1534.

Eler, G. J., \& Jaques, A. E. (2006). O enfermeiro e as terapias complementares para o alívio da dor. Arquivos de Ciências da Saúde da UNIPAR, 10(3), 185190.

Elisabetsky, E., Brum, L. F., \& Souza, D. O. (1999). Anticonvulsant properties of linalool in glutamate-related seizure models. Phytomedicine, 6(2), 107-113.

Fan, W., Huang, F., Wu, Z., Zhu, X., Li, D., \& He, H. (2012). The role of nitric oxide in orofacial pain. Nitric Oxide, 12(1), 32-43.

Ferreira, V. F., \& Pinto. A. C. (2010). A fitoterapia no mundo atual. Química Nova, 1829p.

Guimarães, A. G., Serafini, M. R., \& Quintans-Júnior, L. J. (2014). Terpenes and derivatives as a new perspective for pain treatment: a patent review. Expert Opinion on Therapeutic Patents, 24(3), 243-265.

Holanda Pinto, S. A., Pinto, L. M. S., Guedes, M. A., Cunha, G. M. A., Chaves, M. H., Santos, F. A., \& Rao, V. S. (2008). Antinoceptive effect of triterpenoid alpha, beta-amyrin in rats on orofacial pain induced by formalin and capsaicin. Phytomedicine, 15(8), 630-634.

Hosseinzadeh, H., \& Talebzadeh, F. (2005). Anticonvulsant evaluation of safranal and crocin from Crocus sativus in mice. Fitoterapia, 76(7-8), 722-724.

Hosseinzadeh, H., \& Younesi, H. M. (2002). Antinociceptive and anti-inflammatory effects of Crocus sativus L. stigma and petal extracts in mice. BMC Pharmacology, 2(1), 7-12.

Hosseinzadeh, H., Karimi, G. H., \& Niapoor, M. (2004). Antidepressant effects of Crocus sativus stigma extracts and its constituents, crocin and safranal, in mice. Journal of Medicinal Plants, 3(11), 48-58.

Hosseinzadeh, H., Modaghegh, M. H., \& Saffari, Z. (2009). Crocus sativus L. (Saffron) extract and its active constituents (crocin and safranal) on ischemiareperfusion in rat skeletal muscle. Evidence-based complementary and alternative medicine: eCAM, 6(3), 343-350.

Jia, Y., Gong, N., Li, T. F., Zhu, B., \& Wang, Y. X. (2015). Peptidic exenatide and herbal catalpol mediate neuroprotection via the hippocampal GLP-1 receptor/B-endorphin pathway. Pharmacological Research, 102, 276-285.

Jiang, J., Sehn, Y. Y., Li, J., Lin, Y. H., Luo, C. X., \& Zhu, D. Y. (2015). (+)-Borneol alleviates mechanical hyperalgesia in models of chronic inflammatory and neuropathic pain in mice. European Journal of Pharmacology, 757, 53-58.

Khalilzadeh, E., Hazrati, R., Sayah, G. V., \& Hasannejad, H. (2015). Opioidergic and cholinergic but not nitric oxide pathways are involved in antinociceptive activity of Vitex agnus-castus essential oil in the acute trigeminal model of pain in rat. Asian Journal of Pharmaceutical and Clinical Research, 8(1), 283-286.

Krzyzanowska, A., \& Avendaño, C. (2012). Behavioral testing in rodent models of orofacial neuropathic and inflammatory pain. Behavioral and Brain Sciences, 2(5), 678-697.

Kumari, A., Kumar, V., \& Yadav, S. K. (2012). Nanotechnology: a tool to enhance values of natural products. Trends in Medical Research, 7, $34-42$.

Lappas, C. M., \& Lappas, N. T. (2012). D-limonene modulates T lymphocyte activity and viability. Cellular Immunology, 279(1), 30-41.

Lawson, C. (2009). The role of patents in biodiversity conservation. Nature Biotechnology, 27(11), 994-995.

Lima, D. F., Brandão, M. S., Moura, J. B., Leitão, J. M., Carvalho, F. A., Miúra, L. M., Leite, J. R., Sousa, D. P., \& Almeida, F. R. (2012). Antinociceptive activity of the monoterpene alpha-phellandrene in rodents: possible mechanisms of action. Journal of Pharmacy and Pharmacology, 64(2), $283-293$.

Lima, P. S. S., Lucchese, A. M., Araújo-Filho, H. G., Menezes, P. P., Araújo, A. A. S., Quintans-Júnior, L. J., \& Quintans, J. S. S. (2016). Inclusion of terpenes in cyclodextrins: preparation, characterization and pharmacological approaches. Carbohydrate Polymers, 151, 965-987.

Liu, M., Zhou, L., Chen, Z., \& Hu, C. (2012). Analgesic effect of iridoid glycosides from Paederia scandens (LOUR.) MERRILL (Rubiaceae) on spared nerve injury rat model of neuropathic pain. Pharmacology Biochemistry \& Behavior, 102(3), 465-470.

Liu, R., Zhang, L., Lan, X., Li, L., Zhang, T. T., Sun, J. H., \& Du, G. H. (2011). Protection by borneol on cortical neurons against oxygen-glucose deprivation/reperfusion: involvement of anti-oxidation and anti-inflammation through nuclear transcription factor kappaB signaling pathway. Neuroscience, $176,408-419$

Ma, Y., Zhou, L. L., Yan, H. Y., \& Liu, M. (2009). Effects of extracts from Paederia scandens (LOUR.) MERRILL (Rubiaceae) on MSU crystal-induced rats gouty arthritis. American Journal of Chinese Medicine, 37(4), 669-683.

Moher, D., Liberati, A., Tetzlaff, J., Altman, D. G., The Prisma Group. (2015). Principais itens para relatar Revisões sistemáticas e Meta-análises: A recomendação PRISMA. Epidemiologia e Serviços de Saúde, 24(2), 335-342. 
Nanjundaiah, S. M., Venkatesha, S. H., Yu, H., Tong, L., Stains, J. P., \& Moudgil, K. D. (2012). Celastrus and its bioactive celastrol protect against bone damage in autoimmune arthritis by modulating osteoimmune cross-talk. The Journal of Biological Chemistry, 287(26), 22216-22226.

Nascimento, S. S., Araújo, A. A., Brito, R. G., Serafini, M. R., Menezes, P. P., DeSantana, J. M., Lucca, W., Jr, Alves, P. B., Blank, A. F., Oliveira, R. C., Oliveira, A. P., Albuquerque, R. L., Jr, Almeida, J. R., \& Quintans Júnior, L. J. (2014). Cyclodextrin-complexed Ocimum basilicum leaves essential oil increases Fos protein expression in the central nervous system and produce an antihyperalgesic effect in animal models for fibromyalgia. International Journal of Molecular Sciences, 16(1), 547-563.

Nishijima, C. M., Ganev, E. G., Mazzardo-Martins, L., Martins, D. F., Rocha, L. R., Santos, A. R., \& Hiruma-Lima, C. A. (2014). Citral: A monoterpene with prophylactic and therapeutic antinociceptive effects in experimental models of acute and chronic pain. European Journal of Pharmacology, $736,16-25$.

Nucci, C., Mazzardo-Martins, L., Stramosk, J., Brethanha, L. C., Pizzolatti, M. G., Santos, A. R., \& Martins, D. F. (2012). Oleaginous extract from the fruits Pterodon pubescens Benth induces antinociception in animal models of acute and chronic pain. Journal of Ethnopharmacology, 143(1), 170-178.

Nucci-Martins, C., Martins, D. F., Nascimento, L. F., Venzke, D., Oliveira, A. S., Frederico, M. J., Silva, F. R., Brighente, I. M., Pizzolatti, M. G., \& Santos, A. R. (2015). Ameliorative potential of standardized fruit extract of Pterodon pubescens Benth on neuropathic pain in mice: evidence for the mechanisms of action. Journal of Ethnopharmacology, 175, 273-286.

Oliveira, M. A., Guimarães, A. G., Araújo, A. A. S., Quintans-Júnior, L. J., \& Quintans, J. S. S. (2017). New drugs or alternative therapy to blurring the symptoms of fibromyalgia-a patent review. Expert Opinion on Therapeutic Patents, 27(10), 1147-1157.

Oliveira, S. G. D., Moura, F. R. R., Demarco, F. F., Nascente, P. S., Del Pino, F. A. B., \& Lund, R. G. (2012). An ethnomedicinal survey on phytotherapy with professionals and patients from Basic Care Units in the Brazilian Unified Health System. Journal of Ethnopharmacology, $140(2), 428-437$.

Oliveira, T. V. C., Meneses, D. C. S., Santana, I. C. J., Ferreira, M. E. R., Leite, R. N. O., Carvalho Neto, J. N., Pereyra, B. B. S., Cândido, J. F., Dantas, C. G., Gomes, M. Z., \& Cândido, E. A. F. (2018). Correlation of spasticity with functionality of spastic individuals after physiotherapy treatment associated with herbal medicine derived from Alpinia zerumbet. International Journal of Development Research, 8(1), 18443-18447.

Orhan, I., Küpeli, E., Aslan, M., Kartal, M., \& Yesilada, E. (2006). Bioassay-guided evaluation of anti-inflammatory and antinociceptive activities of pistachio, Pistacia vera L. Journal of Ethnopharmacology, 105(1-2), 235-240.

Otuki, M. F., Ferreira, J., Lima, F. V., Meyre-Silva, C., Malheiros, A., Muller, L. A., Cani, G. S., Santos, A. R., Yunes, R. A., \& Calixto, J. B. (2005). Antinociceptive properties of a mixture of alpha-amyrin and beta-amyrin triterpenes: evidence for participation of protein kinase $\mathrm{C}$ and protein kinase $\mathrm{A}$ pathways. Journal of Pharmacology and Experimental Therapeutics, 313(1), 310-318.

Oussalah, M., Caillet, S., Saucier, L., \& Lacroix, M. (2007). Inhibitory effects of selected plant essential oils on the growth of four pathogenic bacteria: E. coli O157:H7, Salmonella Typhimurium, Staphylococcus aureus and Listeria monocytogenes. Food Control, 18(5), 414-420.

Paris, D., Ganey, N. J., Laporte, V., Patel, N. S., Beaulieu-Abdelahad, D., Bachmeier, C., March, A., Ait-Ghezala, G., \& Mullan, M. J. (2010). Reduction of beta-amyloid pathology by celastrol in a transgenic mouse model of Alzheimer's disease. Journal of Neuroinflammation, $7,17$.

Parmar, S. K., Sharma, T. P., Airao, V. B., Bhatt, R., Aghara, R., Chavda, S., Rabadiya, S. O., \& Gangwal, A. P. (2013). Neuropharmacological effects of triterpenoids. Phytopharmacology, 4(2), 354-372.

Paula-Freire, L. I., Andersen, M. L., Molska, G. R., Köhn, D. O., \& Carlini, E. L. (2013). Evaluation of the antinociceptive activity of Ocimum gratissimum L. essential oil and its isolated active principles in mice. Phytotherapy Research, 27(8), 1220-1224.

Paula-Freire, L. I., Molska, G. R., Andersen, M. L., \& Carlini, E. L. (2016). Ocimum gratissimum essential oil and its isolated compounds (eugenol and myrcene) reduce neuropathic pain in mice. Planta Medica, 82(3), 211-216.

Piccinelli, A. C., Santos, J. A., Konkiewitz, E. C., Oesterreich, S. A., Formagio, A. S., Croda, J., Ziff, E. B., \& Kassuya, C. A. (2015). Antihyperalgesic and antidepressive actions of (R)-(+)-limonene, $\alpha$-phellandrene, and essential oil from Schinus terebinthifolius fruits in a neuropathic pain model. Nutritional Neuroscience, 18(5), 217-224.

Pina, L. T. S., Gouveia, D. N., Costa, J. S., Quintans, J. S. S., Quintans-Júnior, L. J., Barreto, R. S. S., \& Guimarães, A. G. (2017). New perspectives for chronic pain treatment: a patent review (2010-2016). Expert Opinion on Therapeutic Patents, 27(7), 787-796.

Quintans-Júnior, L. J., Araújo, A. A. S., Brito, R. G., Santos, P. L., Quintans, J. S. S., Menezes, P. P., Serafini, M. R., Silva, G. F., Carvalho, F. M. S., Brogden, N. K., \& Sluka, K. A. (2016). $\beta$-caryophyllene, a dietary cannabinoid, complexed with $\beta$-cyclodextrin produced anti-hyperalgesic effect involving the inhibition of Fos expression in superficial dorsal horn. Life Sciences, 149, 34-41.

Quintans-Júnior, L. J., Barreto, R. S. S., Menezes, P. P., Almeida, J. R. G. S., Viana, A. F. S. C., Oliveira, R. C. M., Oliveira, A. P., Gelain, D. P., Lucca Júnior, W., \& Araújo, A. A. S. (2013). $\beta$-Cyclodextrin-complexed (-)-linalool produces antinociceptive effect superior to that of (-)-linalool in experimental pain protocols. Basic \& Clinical Pharmacology \& Toxicology, 113(3), 167-172.

Quintão, N. L. M., Silva, G. F., Antonialli, C. S., Rocha, L. W., Filho, V. C., \& Cicció, J. F. (2010). Chemical composition and evaluation of the antihypernociceptive effect of the essential oil extracted from the leaves of Ugni myricoides on inflammatory and neuropathic models of pain in mice. Planta Medica, 76(13), 1411-1418.

Saberi, M., Rezvanizadeh, A., \& Bakhtiarian, A. (2008). The antiepileptic activity of Vitex agnus-castus extract on amygdala kindled seizures in male rats. Neuroscience Letters, 441(2), 193-196.

Santos, O. J., Barros-Filho, A. K. D., Malafaia, O., Ribas-Filho, J. M., Santos, R. H. P., \& Santos, R. A. P. (2012). Schinus terebinthifolius raddi (anacardiaceae) in the healing process of gastrorraphy in rats. Arquivos Brasileiros de Cirurgia Digestiva (São Paulo), 25(3), 140-146. 
Research, Society and Development, v. 10, n. 5, e6710514606, 2021

(CC BY 4.0) | ISSN 2525-3409 | DOI: http://dx.doi.org/10.33448/rsd-v10i5.14606

Santos-Júnior, L., Oliveira, T. V. C., Cândido, J. F., Santana, D. S., Pereira Filho, R. N., Pereyra, B. B. S., Gomes, M. Z., Lima, S. O., Albuquerque-Júnior, R. L. C., \& Cândido, E. A. F. (2017). Effects of the essential oil of Alpinia zerumbet (Pers.) B.L. Burtt \& R.M. Sm. on healing and tissue repair after partial Achilles tenotomy in rats. Acta Cirúrgica Brasileira, 32(6), 449-458.

Schestatsky, P. (2008). Definição, diagnóstico e tratamento da dor neuropática. Revista do Hospital das Clínicas de Porto Alegre, 28(3), $177-187$.

Silva, K. A. B. S., Paszcuk, A. F., Passos, G. F., Silva, E. S., Bento, A. F., Meotti, F. C., \& Calixto, J. B. (2011). Activation of cannabinoid receptors by the pentacyclic triterpene $\alpha, \beta$-amyrin inhibits inflammatory and neuropathic persistent pain in mice. Pain, 152(8), 1872-1887.

Siqueira-Lima, P. S., Silva, J. C., Quintans, J. S.S., Antoniolli, A. R., Shanmugam, S., Barreto, R. S. S., Santos, M. R. V., Almeida, J. R. G. S., Bonjardim, L. R., Menezes, I. R.A., \& Quintans-Júnior, L. J. (2017). Natural products assessed in animal models for orofacial pain - a systematic review. Revista Brasileira de Farmacognosia, 27(1), 124-134.

Sousa, D. G., Sousa, S. D. G., Silva, R. E. R., Silva-Alves, K. S., Ferreira-da-Silva, F. W., Kerntopf, M. R., Menezes, I. R. A., Leal-Cardoso, J. H., \& Barbosa, R. (2015). Essential oil of Lippia alba and its main constituent citral block the excitability of rat sciatic nerves. Brazilian Journal of Medical and Biological Research, 48(8), 697-702.

Stojković, D., Soković, M., Glamočlija, J., Džamić, A., Ćirić, A., Ristić, M., \& Grubišić, D. (2011). Chemical composition and antimicrobial activity of Vitex agnus-castus L. fruits and leaves essential oils. Food Chemistry, 128(1), 1017-1022.

Upton, R. (2001). Chaste Tree Fruit, Vitex Agnus-Castus: Standards of Analysis, Quality Control, and Therapeutics, Santa Cruz, CA. American Herbal Pharmacopoeia: Santa Cruz, CA.

Venkatesha, S. H., Yu, H., Rajaiah, R., Tong, L., \& Moudgil, K. D. (2011). Celastrus-derived celastrol suppresses autoimmune arthritis by modulating antigen-induced cellular and humoral effector responses. The Journal of Biological Chemistry, 286(17), 15138-15146.

Wang, S., Zhang, D., Hu, J., Jia, Q., Xu, W., Su, D., Song, H., Xu, Z., Cui, J., Zhou, M., Yang, J., \& Xiao, J. (2017). A clinical and mechanistic study of topical borneol-induced analgesia. EMBO Molecular Medicine, 9(6), 802-815.

Wang, W., Ha, C., Lin, T., Wang, D., Wang, Y., \& Gong, M. (2018). Celastrol attenuates pain and cartilage damage via SDF-1/CXCR4 signalling pathway in osteoarthritis rats. Journal of Pharmacy and Pharmacology, 70(1), 81-88.

Wang, Y., Zhang, R., Xie, J., Lu, J., \& Yue, Z. (2014). Analgesic activity of catalpol in rodent models of neuropathic pain, and Its spinal mechanism. Cell Biochemistry and Biophysics, 70(3), 1565-1571.

Yang, L., Li, Y., Ren, J., Zhu, C., Fu, J., Lin, D., \& Qiu, Y. (2014). Celastrol attenuates inflammatory and neuropathic pain mediated by cannabinoid receptor type 2. International Journal of Molecular Sciences, 15(8), 13637-13648. 